\title{
EP-193
}

\section{Readmissions after pancreaticoduodenectomy}

\author{
Krishna RAO GURANA*, Vinay KUMAR KAPOOR, Rajan SAXENA, Ashok KUMAR GUPTA, Anu BEHARI, \\ Rajneesh K SINGH, Supriya SHARMA, Ashok, Ashish SINGH, Rahul
}

Department of Surgical Gastroenterology, Sanjay Gandhi Post Graduate Institue of Medical Sciences, Lucknow, India

Introduction: Readmissions indicate complexity of procedure, disease progression and care provided. Pancreatoduodenectomy (PD) is a complex procedure with high morbidity and mortality. Though mortality of PD has reduced, high morbidity remains cause for concern. Knowledge of readmissions enlightens us regarding postprocedure complications and disease progression and help improve standard of care.

Methods: Patients who underwent PD 1991 to 2020 were reviewed retrospectively.

Results: 187 (19\%) out of 1007 cases required readmission - common causes of readmission were sequel of surgery 93 (50\%) and recurrence 59 (33\%). 40 (67\%) out of 59 recurrence were ampullary cancer, 8 (14\%) were pancreatic head cancer. $44 / 187$ (24\%) required readmission within 30 days - 38 (86\%) for sequel of surgery - intraabdominal collection 10 (27\%) and bleeding 6 (16\%). 13 (34\%) required intervention - percutaneous drainage of collection 5, upper GI endoscopy 4, angiography and intravascular procedure 2, surgical drainage under local anaesthesia 2 . There was one mortality and 13 (34\%) patients suffered morbidity of Clavien Dindo grade 3 and above. 32 (73\%) were ampullary and 5 (11\%) were benign. 41 (22\%) patients required readmission 30 to 90 days - 15 (36\%) for sequel of surgery and 4 (9\%) for recurrence. 3 (7\%) required intervention. $102(55 \%)$ patients required readmission after 90 days - 43 (42\%) with recurrence and 24 (24\%) for surgical sequel. 20 (20\%) required intervention - $8(40 \%)$ surgical procedure and $12(60 \%)$ percutaneous. Two patients died in the hospital and 4(with recurrence) were expected deaths. 71 (70\%) had ampullary carcinoma and 9 (9\%) had pancreatic head cancer, 9 (9\%) had benign pathology.

Conclusions: Pancreatoduodenectomy is a complex procedure, many patients require readmission - early for sequel of surgery and late for recurrence. 